\title{
Activation of peripheral neuronal FLT3 promotes exaggerated sensorial and emotional pain-related behaviors facilitating the \\ transition from acute to chronic pain
}

Adrien Tassou ${ }^{1,2}$, Maxime Thouaye ${ }^{1,2}$, Damien Gilabert ${ }^{1,3}$, Antoine Jouvenel ${ }^{1,2}$, Jean-Philippe Leyris ${ }^{2,6}$, Corinne Sonrier ${ }^{1,2,6}$, Lucie Diouloufet ${ }^{1,2,6}$, Ilana Mechaly ${ }^{1,2}$, Myriam Chentouf ${ }^{1,5}$, Madeline Neiveyans ${ }^{1,5}$, Martine Pugnière ${ }^{1,5}$, Pierre Martineau ${ }^{1,5}$, Bruno Robert $^{1,5}$, Xavier Capdevila $^{4}$, Jean Valmier ${ }^{1,2}$, Cyril Rivat $^{1,2^{*}}$

Adresses : ${ }^{1}$ Univ Montpellier, Montpellier, France ; ${ }^{2}$ Inserm U-1298, Institut des Neurosciences de Montpellier, Montpellier, France ; ${ }^{3}$ CNRS UMR 5203, Institut de Génomique Fonctionnelle, Montpellier, France $;{ }^{4}$ Département d'anesthésiologie, Hôpital Universitaire Lapeyronie, Montpellier, France ; 5 IRCM, INSERM U1194, ICM, Montpellier, F-34298, France, ${ }^{6}$ BIODOL Therapeutics, Cap Alpha, Clapiers, France.

\$ current address: University of North Carolina at Chapel Hill, Neuroscience center, Mary Ellen Jones, building, Chapel Hill, NC 27599, USA

* Corresponding author: cyril.rivat@,umontpellier.fr

Conflict of interest statement: The authors declare no conflict of interests 


\section{Abstract}

Background. Acute pain events have been associated with persistent pain sensitization of nociceptive pathways increasing the risk of transition from acute to chronic pain. However, it is unclear whether injuryinduced persistent pain sensitization can promote long-term mood disorders. The receptor tyrosine kinase FLT3 is causally required for peripheral nerve injury-induced pain chronification, questioning its role in the development of pain-induced mood alterations.

Methods. In a model of paw incisional pain, mice underwent single (SI) or double incision (DI) and went through behavioral and molecular phenotyping with the evaluation of both sensorial and emotional pain components. The role of FLT3 was then investigated either by inhibition using transgenic knock-out mice and functional antibodies or by activation with FLT3 ligand (FL) administrations.

Results. DI mice showed significant anxiodepressive-like and spontaneous pain behaviors while SI mice did not. DI also promoted and extended mechanical pain hypersensitivity compared to SI. This emotional and sensorial pain exaggeration correlated with a potentiation of spinal microglial activation after DI versus SI. Intrathecal minocycline, a microglial inhibitor, specifically reversed DI induced-mechanical hypersensitivity. Finally, FL injections in naive animals provoked mechanical allodynia and anxiodepressive-like disorders concomitant with a significant microglial activation while FLT3 inhibition blunted the development of persistent pain and depression after DI.

Conclusions. Our results show for the first time that the repetition of a peripheral lesion facilitates not only exaggerated nociceptive behaviors but also anxiodepressive disorders. The inhibition of FLT3 could become a promising therapy in the management of pain sensitization and related mood alterations. 


\section{Introduction}

Understanding the transition from acute to chronic pain remains an important challenge to better manage pain in patients (1). This is specifically true in the context of surgery where chronic post-surgical pain (CPSP) is a significant debilitating and under-evaluated disease affecting several millions of people each year in the world (2-5). Pre-existing pain is one major risk factor as it profoundly modifies patient's internal homeostasis altering the degree of pain sensitization of the patient (6-8). Hence, sensitization after sensorial trauma appears to be a crucial event in the development of chronic pain by amplifying signaling in previously affected nociceptive pathways (9). Animal models called "hyperalgesic priming" are classically used to study the neuroplasticity underlying persistent pain sensitization (10-18). These models consist in performing an initial nociceptive stimulation that induces a prolonged period of susceptibility to exaggerated sensitization after a subsequent stimulation. This phenomenon has been named latent pain sensitization (19) and its mechanisms have been further studied (20-22). The clinical observation that the incidence of chronic pain after injury is largely associated with pre-injury pain status (6) suggests that the maintenance of latent sensitization may facilitate the transition from acute to persistent pain. Mood disorders such as depression and anxiety are frequently observed in patients suffering from chronic pain. It has been reported that at least $50 \%$ of chronic pain patients experience a major depressive disorder (23), making depression and chronic pain together a major comorbidity (24-26). Similarly, persistence of mood disorders becomes a maladaptive process maintained by neuropathological mechanisms that can aggravate the sensory abnormalities of chronic pain $(27,28)$. Although this comorbidity is clinically well established, the underlying pathophysiological mechanisms remain poorly understood. It has been proposed that depression is a consequence of the presence of chronic pain $(29,30)$ and that peripheral nociceptive inputs may act as a trigger for mechanisms that leave predisposed individuals at increased risk of anxiodepressive disorders. Thus, we hypothesize that peripheral nociceptive inputs may generate sustained neurochemical alterations (latent sensitization) leading to affective disorders after subsequent re-exposure to nociceptive stimuli as 
shown with exaggerated pain hypersensitivity in hyperalgesic priming models. To date, the affective dimension has never been studied in animal models of hyperalgesic priming. Importantly, this incomplete characterization has impeded the understanding of the role of peripheral nociceptive inputs in the development of affective disorders. Hence, to test our hypothesis, we characterize a model of repeated hind paw injuries where the animals underwent two hind paw surgery 7 days apart, by studying both sensorial and affective alterations with behavioral and molecular approaches. This allows us to determine whether repeated exposure to peripheral noxious stimuli can trigger both exaggerated pain hypersensitivity and maladaptive processes leading to anxiodepressive disorders.

Recently, we reported the expression of the fms-like tyrosine kinase 3 receptor (FLT3) in the peripheral nervous system (31) and its implication in the development and maintenance of chronic neuropathic pain via its ligand, the cytokine FL (FLT3 Ligand). Originally identified as part of the hematopoietic system, we showed that FLT3, expressed in rodent DRG neurons, induces long-term modifications in DRG neurons, leading to neuronal hyperexcitability giving rise to neuropathic pain symptoms. In contrast, the genetic and pharmacological inhibition of FLT3 prevents, but also reverses, hyperexcitability and pain-related behaviors associated with nerve injury. These results strongly highlight the key role of FLT3 in the development of long-term neuropathic pain sensitization. Although its role in the sensorial component of neuropathic pain is now well established, we are still lacking evidence about the extent to which peripheral FLT3 can be involved in the central consequences of latent sensitization, especially in the affective dimension of pain. Hence, in a second objective we reveal for the first time, a FLT3 dependent peripheral sensitization that triggers both exaggerated nociceptive and affective disorders after repeated surgical injury. 


\section{Methods and materials}

Detailed material and methods are provided in Supplement 1. All the procedures were approved by the French Ministry of Research (authorization \#1006). Animals were maintained in a climate-controlled room on a $12 \mathrm{~h} \mathrm{light/dark} \mathrm{cycle} \mathrm{and} \mathrm{allowed} \mathrm{access} \mathrm{to} \mathrm{food} \mathrm{and} \mathrm{water} \mathrm{ad} \mathrm{libitum.} \mathrm{Male} \mathrm{and} \mathrm{female} \mathrm{mice} \mathrm{were} \mathrm{first}$ considered separately in behavioral procedures. Both sexes showed mechanical hypersensitivity of same intensity after intrathecal FL injection and after surgery and were similarly affected by FLT3 deletion (ANOVA followed by Bonferroni's test, $\mathrm{n}=8$ for both sexes and genotypes for each experiment). Thereafter, experiments were performed only on male mice. Different approaches including mainly behavior, immunohistochemistry, real time polymerase chain reaction, the development and production of humanized antibody were used to evaluate the sensorial and emotional alterations produced by repeated injuries and the contribution of FLT3 in these phenomena.

\section{Results}

Chronic pain is characterized by a variety of symptoms including mechanical allodynia, thermal hypersensitivity, spontaneous pain and a high susceptibility to develop depression. Therein, we first determined whether a mouse model of pain sensitization triggered by repeated hindpaw incision produced alteration of emotional-related behaviors associated with exaggerated sensory behaviors. For this purpose, mice were assessed in a set of behavioral tests to evaluate emotional (ST: Splash Test; NSF: Novelty Suppressed Feeding test; FST: Forced Swim Test) and sensorial (VF: Von Frey filament test; HG: Hargreaves test; CPP: Conditioned place preference test) components after repeated acute injuries.

SI (Single Incision, one incision on the left hindpaw) (32) and DI (Double Incision, first incision on the left hindpaw and second one on the right hindpaw) models were compared and tested at three different time points after surgery: the following days after surgery ( $1^{\text {st }}$ week post-incision $)$, just after nociceptive 
recovery $\left(2^{\text {nd }}\right.$ week post-incision $)$ and 1 month after incision. The first week after surgery, the rotarod test did not reveal any difference between Sham, SI and DI mice indicative of an absence of motor impairment in animals with SI or DI (Fig. S1A). SI and DI mice responded to the NSF with an increased latency to eat compared to Sham mice while only DI mice responded to ST and FST with a decreased grooming behavior and immobility, respectively (Fig. S1B-D). During the $2^{\text {nd }}$ week after surgery, no difference between Sham mice and SI mice were found in all the tests while DI mice showed sustained anxiodepressive-like behaviors (Fig. 1A-C). One month after surgery, all the experimental groups returned to Sham values (Fig. S1E-G). These results are resumed in Fig. 1D. Decreased neurogenesis at the level of the dentate gyrus has been associated with depression in humans $(33)$ and animal $(34,35)$ studies. We therefore hypothesized that DIinduced anxiodepressive-like behaviors could decrease the rate of newborn neurons in this region as evaluated with Brdu staining. Brdu stained neurons (Brdu/NeuN colocalisation) were substantially decreased in DI mice 7 days after the surgery compared to SI and Sham mice (Fig. 1E-F) supporting the development of mood disorder in DI mice.

We also tested the effect of SI vs. DI on sensory thresholds and ongoing pain. Six hours after the first surgery, mice mechanical thresholds as measured by the VF test strongly decreased up to ten-fold in ipsilateral hindpaw (left hindpaw) indicating the appearance of mechanical allodynia (Fig. 2A). Mice recovered a normal mechanical threshold after 7 days, and mechanical threshold was unchanged in the SI contralateral hindpaw (right hindpaw). By contrast to the first incision, the second incision induced a robust decrease of mice withdrawal thresholds at the ispilateral hindpaw (right hindpaw) and at the contralateral hindpaw (left hindpaw; Fig. 2A). As reported in a previous work (36), recovery to a normal threshold of both hindpaws was delayed of 7 days compared to the first incision, with a full recovery at day 22 . Area Under Curve (AUC) that represents the period of mechanical hypersensitivity observed the days after incision on the ipsilateral paw further confirmed that DI mice exhibited exaggerated mechanical pain hypersensitivity compared to SI mice (Fig. 2B). Mice also displayed prolonged thermal pain hypersensitivity after the second incision compared to the first one, as evaluated with the HG test (Fig. 2C). Therefore, we 
used CPP experiments 3 days after surgery (SI or DI) to assess spontaneous pain behaviors (37). Only the DI experimental group developed a place preference for the clonidine-paired chamber (Fig. 2D) indicative of significant ongoing pain in DI animals.

We then studied the peripheral nervous system alterations induced by the procedures through retrograde tracing of injured peripheral nerves. First, we administered Fluorogold to retrogradely label the DRG neurons affected by incision. At Day 7 (D7), most of the Fluorogold positive cells were found in L4 (DI: 27\%; SI: 22\%), few in L5 (DI, SI: 3\%) and 0\% in L6 DRGs (Fig. S2A-B). Co-staining of ATF3 and CSF1, two important molecular factors specifically expressed after neuronal stress or injury $(38,39)$ was realized at D7 (Fig. S2 C-E). Similarly, most of ATF3 and CSF1 positive neurons were found in L4 (10$15 \%)$, few in L5 ( $3 \%)$ and $0 \%$ in L6 DRGs. No differences were found between SI and DI mice suggesting that these peripheral alterations are unlikely responsible for the exaggerated pain-related behaviors after DI. We next examined, using real time quantitative PCR, the expression of different markers of pain sensitization in the dorsal horn of the spinal cord. Interestingly, the nerve injury factors Atf3 and Sprrla mRNA in ipsilateral dorsal horn spinal cord were strongly increased after incision, with a potentiation in DI as compared to SI (Fig. S3). Because we found no changes in the relative expression of nerve-injury related proteins in DRG neurons and since pain-related markers mRNAs were increased in the spinal cord of DI compared to SI mice, we then investigated spinal cells alterations through the labeling of peptidergic neurons (CGRP) and glial cells (Iba1 and GFAP). CGRP staining was found increased in the injured side of SI dorsal horns compared to Sham. DI increased CGRP staining on both sides, without any significative difference between SI and DI (Fig. S4A-C). GFAP staining in astrocytes was found unchanged at D3 and D7 compared to control group (Fig. S4D-F). Markers associated with microglial activation were also quantified using RTqPCR (Fig. 2E). Levels of Cd11b, Iba1, Csf1 and Csf1r mRNA were all found higher after incision (SI or DI) but no differences were found between SI and DI conditions. By contrast, at D7, microglial Iba1 staining density was found increased compared to Sham (SI vs. Sham: x 1.8) but also potentiated in DI compared to SI mice (DI vs. Sham: x 3.2; DI vs. SI: x 1.6) (Fig. 2F-G). To further show the implication of microglia in 
the exaggeration of nociceptive behaviors, we then treated Sham, SI and DI mice with a single intrathecal injection of microglial inhibitors (40), minocycline $(300 \mu \mathrm{g} / 5 \mu \mathrm{l} / \mathrm{animal})$ at $\mathrm{D} 3$, when incision-induced pain hypersensitivity was still observed. Strikingly, minocycline restored mechanical thresholds to normal values only in DI mice, whereas it had no effect in Sham or SI mice (Fig. 2H). Similar results were obtained with GW2580 (1 $\mu \mathrm{g} / 5 \mu \mathrm{l} / \mathrm{animal})$, another microglial inhibitor (41), when intrathecally administered at the time of the surgeries (Fig. S5A-B). Interestingly, mice treated with GW2580 displayed enhanced grooming behaviors during the ST and reduced latency to eat during the NSF compared to untreated mice suggesting that the inhibition of spinal microgliosis may also have an impact in the development of mood disorders (Fig. S5D-F). Collectively these data show that surgery repetition prolongs mechanical/thermal hypersensitivity and induces spontaneous pain behavior supported by potentiation of microglial activation in the spinal cord that seems responsible not only for mechanical hypersensitivity after DI but also mood disorders.

Our results here identify the overexpression of neuronal stress-related molecular markers (Sprr1a and Atf3) after DI suggesting a higher neuropathic component in DI compared to SI model. We recently showed that peripheral FLT3 inhibition prevents the development of neuropathic pain (31). Hence, we questioned the implication of FLT3 receptors in the development of exaggerated pain-related behaviors by assessing both sensorial and emotional behaviors after DI in Flt3 Knock-out (Flt3 ${ }^{-/ /}$) female and male mice. In the VF test, basal mechanical threshold was not affected by FLT3 silencing, as already described (31). After surgery, Flt3 $3^{-/-}$male mice displayed a faster recovery (Fig. 3A-C) along with reduced mechanical pain hypersensitivity to both the first and the second incision compared to male Flt3 Wild Type $\left(\mathrm{Flt}^{+/+}\right)$mice. Similar results were observed in female Flt $3^{-/-}$mice (Fig. S6D-E). CPP experiments also confirm the presence of a place preference for the clonidine-paired chamber in the DI Flt $3^{+/+}$group. This place preference was totally prevented in DI Flt3 ${ }^{-/-}$animals (Fig. 3D). To check for a potential role of FLT3 in basal mood modulation, we compared anxiodepressive-like behaviors of $\mathrm{Flt}^{-/-}$with $\mathrm{Flt}^{+/+}$animals in naïve condition (Fig. S6A-C). No differences were observed between Flt $^{-/-}$and Flt $^{+/+}$mice in the ST and NSF but Flt3 $3^{-/-}$ 
already displayed decreased immobility duration in the FST. Afterward, the effects of FLT3 silencing on DI-induced anxiodepressive like behaviors were evaluated. Because these behaviors require motor functions, locomotor activity and motor coordination were systematically assessed in all tested animals (Fig. 3E; Fig. S6F) and were found unchanged. Flt3 $3^{-/}$animals showed no significant differences with WT mice in NSF but were significantly less immobile in the FST and presented increased grooming in the ST (Fig. 3F-H). At the molecular level, Ibal staining in the dorsal horn of WT animals was increased whereas no change was found in Flt3 $^{-/-}$animals after DI (Fig. 3I-L).

To evaluate whether local FLT3 activation alone can induce both sensorial and emotional alterations as displayed in the DI model, mice received intrathecal injections of FL (50ng/5 $\mu 1 /$ animal) and were tested. As previously shown, a single injection led to decreased mechanical threshold for at least 2 days (Fig. 4A). Strikingly, repeated intrathecal FL (2 weeks, linj/3days) failed to impair motor coordination but induced decreased grooming behavior, increased latency to eat and immobility duration on the ST, NSF and FST compared to vehicle-treated animals, respectively (Fig. 4B-E). All behaviors were significantly modified in all tests after repeated FL treatment, mimicking features of the DI model. At the cellular level, Iba1 staining was found quantitatively enhanced after repeated FL vs vehicle injections (Fig. 4F-G), confirming its effect on central changes in the spinal cord. To rule out a possible implication of FLT3 expression in microglia cells after surgery, we performed Flt3 in situ hybridization of spinal cord from the CX3CR $1^{\text {EGFP }}$ mouse line, in which EGFP is expressed in CX3CR1+ cells, known as macrophages (including microglia). Our data revealed the absence of colocalization between Flt3 mRNA and CX3CR1 (Fig. S7). These data suggest that the activation of microglia is not mediated by the microglia itself but likely through the activation of the peripheral FLT3 promoting central pain sensitization, causing emotional and sensorial alterations.

Lastly, we developed cross-reacting antibodies specifically directed against both human and mice FLT3. Functional antibodies have the interesting property to not cross the blood brain barrier and could be an advantageous tool for questioning peripheral FLT3 functions, and potentially treat postoperative pain in human. Among all the antibodies produced, the mAbA3 antibody was found to present high affinity for both 
receptors (Fig. 5A-B, Fig. S8). Moreover, Homogeneous Time Resolved Fluorescence (HTRF) signals of FLT3-expressing RS4-11 cells in the absence or presence of FL was strongly reduced in a dose dependent manner after exposure to mAbA3 (Fig. 5C-E). Because we know the therapeutic benefits of inhibiting FLT3 in neuropathic pain models, in vivo efficacy of the antibody was evaluated in the Chronic Constriction Injury (CCI) of the sciatic nerve model of neuropathic pain. Single or repeated (Fig. 6A, C) systemic injections of mAbA3 totally blocked mechanical pain hypersensitivity evaluated on the injured hindpaw. Inhibition of mechanical hypersensitivity started at a dose of $50 \mu \mathrm{g} /$ animal of mAbA3 (Fig. 6B). We next tested the efficacy of mAbA3 in animals that underwent repeated incisions. A single preventive systemic injection of $200 \mu \mathrm{g}$ of mAbA3 during the first incision strongly accelerated recovery to a basal mechanical threshold after both the first and the second incision on the ipsilateral hindpaw (Fig. S9A, B). Since a single injection was not able to block CPSP induced anxiodepressive like behaviors (Fig. S9D-F), several injections were made after the first incision ( 1 injection every 2 days until the second incision). Repeating preventive injections of mAbA3 not only reduced pain hypersensitivity but also totally prevented the development of anxiodepressive-like behaviors compared to vehicle-treated mice (Fig. 6D-H). 


\section{Discussion}

Our results show that the repetition of surgical incision (DI) leads to sustained depressive-like behaviors along with the exaggeration of nociceptive behaviors and the appearance of spontaneous pain recapitulating the different features of chronic pain in humans (42). These behaviors are associated with microglial activation in the spinal cord dorsal horn and decreased neurogenesis in the hippocampus supporting central alterations after DI. We report for the first time the implication of FLT3 in sustained pain sensitization produced by repeated injury. Strikingly, FLT3 activation alone is sufficient to recapitulate microglial activation and anxiodepressive-like behaviors observed after DI. Conversely, FLT3 deletion leads to the complete prevention of DI-related behavioral and molecular modifications. Finally, we developed an innovative therapeutic tool represented by the FLT3 human antibody (mAbA3) targeting both human and murine FLT3. Treatment with FLT3 antibodies not only blocked exaggerated pain behaviors produced by repeated surgical procedure but also the subsequent depressive-like behaviors.

To better examine the mechanisms involved in pain chronification after acute injury, we used an experimental procedure that consists of repeating a surgical incision on the opposite hindpaw of the same animals 7 days apart (36). Here, our main objective was to determine whether repeated acute injury may produce pain sensitization peripherally and centrally. To address this question, we characterized the differences observed between SI and DI at the behavioral and molecular levels. As previously reported, the pain hypersensitivity produced by the second surgery is largely enhanced compared to the one produced by the first surgery as it takes 7 days for the pain hypersensitivity to resolve after SI compared to 14 days after DI. Our data suggest that surgery, like inflammation, may produce a sustained sensitized state leading to increased vulnerability to develop persistent pain when sensitized individuals are challenged with subsequent nociceptive stimuli leading to exaggerated nociceptive behaviors $(12,43)$. However, to our knowledge no study has evaluated the impact of such a repetition on the development of emotional disorders. Our data show for the first time that DI not only produces exaggerated pain behaviors but also leads to 
sustained anxiodepressive-like behaviors starting after the second injury and lasting 3 weeks post-injury. Of note, behavioral alterations are associated with reduced hippocampal neurogenesis which is consistent with the depressive phenotype described in both rodents and humans (44-47). Pre-clinical pain models already reported the existence of depressive-like behaviors that usually develop in 5-7 weeks after nerve injury (4850). These models allow to identify the neuronal circuits supporting comorbidity of persistent pain and mood disorders $(51,52)$. Our data shows that DI model is a very attractive model for evaluating the characteristics of peripheral and central processes induced by nociceptive inputs leading to fast and sustained pain and mood sensitization.

As already proposed, the exaggeration of mechanical pain hypersensitivity produced by the second surgery performed in the non-operated hindpaw strongly suggests that pain sensitization is mainly affecting the central nervous system (43). This is further supported by the fact that we were unable to find a marker (ATF3 and CSF1) differentially expressed in DI compared to SI in DRG. Furthermore, retrograde labelling experiments highlight the small proportion of neurons affected by incision, mainly located in L4 DRGs. We then evaluated potential modifications in the spinal cord of these animals. We show that Iba1 immunoreactivity is significantly enhanced after DI compared to SI or control. The curative inhibition of microglial activation via minocycline or GW2580 leads to a quick recovery of pain sensitivity only after DI. Our observation agrees with previous data reporting that primed spinal microglia by neonatal incision is involved in the persistent pain in re-incised adult rat (53). Interestingly, the microglial inhibition via G2580 also reduces anxiodepressive-related behaviors. This indicates that spinal microglial overactivation produced by repeated incisions promotes both the sensorial and emotional alterations caused by DI. Microglia-related genes (CdIlb, Ibal, Csfl, Csflr) were all increased after incision, without any difference between SI and DI conditions suggesting that DI may likely induce post-transcriptional expression modifications rather than transcriptional alterations. Concerning astroglial activation, GFAP expression was not different in the spinal cord after SI or DI, as opposed to what has been reported (54). We can arguably propose that astrogliosis potentially appears later than the investigated timeframe. Furthermore, these 
observations were only made in rats. Altogether, our results show the strong involvement of peripheral neuron/ central microglia interactions, an important mechanism of persistent central pain sensitization, in the exaggeration of nociceptive behaviors, as it has been already shown in other chronic pain models, especially after neuropathic pain (51). We further report that spinal microglia activation is also necessary in peripheral injury-induced depression-related behaviors.

In neuropathic pain models, microgliosis depends on CSF1 release by sensory neurons from DRG (39) that then reaches the spinal cord to activate microglial CSF1R. Our data emphasize a neuropathic-like component in the SI and DI models through the discovery of a peripheral CSF1 activation that is totally absent in an inflammatory pain model (55). This activation was associated with enhanced ATF3 expression in sensory neurons. Additional studies will be necessary to determine which mechanisms are critical for the over-activation of microglia after DI. Finally, the enhanced spinal expression of Atf3 and Sprrla mRNAs, two markers of nerve injury $(38,56)$ confirms the strong neuropathic component in our models. Of note, the increased expression of these markers in DI vs. SI suggests a stronger neuropathic component in DI compared to SI supporting the clinical observation that persistent post-surgical pain is partially neuropathic in nature (57).

In the continuity of our previous work showing the involvement of FLT3 in neuropathic pain and considering the important neuropathic contribution in these models, we then evaluated the effects of FLT3 inhibition and activation in incision-induced central sensitization. Absence of Flt3 expression in null mutant mice leads to faster recovery of pain hypersensitivity after incision, and to a total prevention of spontaneous pain and anxiodepressive-like behaviors. Beneficial behavioral effects of FLT3 silencing are common to both males and females. Spinal microgliosis activation is also reduced in Flt3 $3^{-/-}$animals. Interestingly, FL alone can activate microglia in the dorsal horn of the spinal cord and to recapitulate many aspects of pain sensitization exhibited in DI. The effect of FL seems to be neuron dependent since Flt3 mRNA is absent in CX3CR1 positive cells. Moreover, since Flt3 mRNA is totally absent in the superficial dorsal horn layers of the spinal cord, it is tempting to speculate that the intrathecal injection of FL leads to the activation of 
peripheral FLT3. Altogether, our data suggest that FLT3 expressed in the DRG can produce persistent central pain sensitization via spinal microgliosis. We then developed functional antibodies (mAbA3) in order to reach high affinity and specificity for both mouse and human FLT3 using phage-display and a scFv synthetic library $(58,59)$. Our data show that a single injection of mAbA3 is sufficient to prevent surgeryinduced exaggerated pain hypersensitivity but insufficient to prevent the development of surgery-induced anxiodepressive disorders. We then considered the administration of repeated antibodies starting before the first surgery and repeated every two days until the second surgical challenge. The preventive use of mAbA3 also largely decreases the time of recovery and totally hinders the development of anxiodepressive disorders. These results clearly support the role of peripheral neuronal FLT3 in the development of pain sensitization after repeated injury as the crossing of functional antibodies into the central nervous system is impeded by the blood-brain barrier. Remarkably, we discovered that FLT3 is causally required for the pro-nociceptive effects of microglia. This important finding not only illuminates the neurobiology of pain and microglia function, but also emphasize the relevance of developing therapeutics, like functional antibodies targeting FLT3 for the treatment of chronic pain.

In conclusion, we demonstrate that repeated peripheral incisions lead both to sensorial and emotional alterations through central pain sensitization. These alterations correlate with spinal microglia over-activation which is responsible for DI-induced mechanical hypersensitivity. Increased expression of axonal regeneration genes and markers of neuropathy unveil the strong neuropathic component in our models, particularly in DI. Our study supports peripheral FLT3 as an important upstream neuronal modulator of spinal microgliosis involved in central pain sensitization leading to exaggerated pain-related behaviors and anxiodepressive disorders. Consequently, targeting FLT3 via functional antibodies against both human and mice FLT3 efficiently alleviates sensitization. Collectively, these data open new avenues for the management of the prevention of pain sensitization and chronification. 


\section{Author contribution}

CR, JV designed the project; AT, DG, IM, MT, AJ, CS, LD, JPL did the experiments; MC, MN, BR, PM generated mAbA3; AT, DG analyzed the data; JV, CR provided resources; XC, CR directed the work; AT, CR and JV wrote the manuscript.

\section{Acknowledgments and Disclosure}

This work was supported by the University of Montpellier and grants from the READYNOV Région Occitanie and European community program, SFETD (Gisèle Guilbaud 2019 price), CBS2 doctoral school and BIODOL therapeutics. We are grateful to F. Perrin for providing CX3CR1 ${ }^{\mathrm{EGFP}}$ mouse line, to B. Pau to help for designing FLT3 antibody strategy, to P. Sokoloff, P. Carroll and I. Yalcin for constructive comments on the manuscript. MAbA3 was produced by GenAc platform funded by the French National Research Agency under the program "Investissements d'avenir" Grant Agreement LabEx MAbImprove: ANR-10LABX-53 and by the SIRIC Montpellier-Cancer under Grant Agreement "INCa-DGOS-Inserm 6045. 


\section{Bibliography}

1. Price TJ, Gold MS (2018): From mechanism to cure: Renewing the goal to eliminate the disease of pain. Pain Medicine (United States). https://doi.org/10.1093/pm/pnx108

2. Richebé P, Capdevila X, Rivat C (2018): Persistent Postsurgical Pain: Pathophysiology and Preventative Pharmacologic Considerations. Anesthesiology. https://doi.org/10.1097/ALN.0000000000002238

3. Fregoso G, Wang A, Tseng K, Wang J (2019): Transition from acute to chronic pain: Evaluating risk for chronic postsurgical pain. Pain Physician 22: 479-488.

4. Macrae WA (2008): Chronic post-surgical pain: 10 Years on. Br J Anaesth 101: 77-86.

5. Montes A, Roca G, Sabate S, Lao JI, Navarro A, Cantillo J, Canet J (2015): Genetic and Clinical Factors Associated with Chronic Postsurgical Pain after Hernia Repair, Hysterectomy, and Thoracotomy: A Two-year Multicenter Cohort Study. Anesthesiology 122: 1123-1141.

6. McGreevy K, Bottros MM, Raja SN (2011): Preventing chronic pain following acute pain: Risk factors, preventive strategies, and their efficacy. Eur J Pain Suppl. https://doi.org/10.1016/j.eujps.2011.08.013

7. Kehlet H, Jensen TS, Woolf CJ (2006): Persistent postsurgical pain: risk factors and prevention. Lancet. https://doi.org/10.1016/S0140-6736(06)68700-X

8. Aasvang EK, Gmaehle E, Hansen JB, Gmaehle B, Forman JL, Schwarz J, et al. (2010): Predictive risk factors for persistent postherniotomy pain. Anesthesiology 112: 957-969.

9. Glare P, Aubrey KR, Myles PS (2019): Transition from acute to chronic pain after surgery. The Lancet. https://doi.org/10.1016/S0140-6736(19)30352-6

10. Dina OA, McCarter GC, De Coupade C, Levine JD (2003): Role of the sensory neuron cytoskeleton in second messenger signaling for inflammatory pain. Neuron 39: 613-624.

11. Parada CA, Reichling DB, Levine JD (2005): Chronic hyperalgesic priming in the rat involves a novel interaction between cAMP and PKC $\varepsilon$ second messenger pathways. Pain 113: 185-190.

12. Reichling DB, Levine JD (2009): Critical role of nociceptor plasticity in chronic pain. Trends in Neurosciences. https://doi.org/10.1016/j.tins.2009.07.007

13. Bogen O, Alessandri-Haber N, Chu C, Gear RW, Levine JD (2012): Generation of a pain memory in the primary afferent nociceptor triggered by PKC $\varepsilon$ activation of CPEB. J Neurosci 32: 2018-2026.

14. Moy JK, Khoutorsky A, Asiedu MN, Black BJ, Kuhn JL, Barragán-Iglesias P, et al. (2017): The MNK-eIF4E signaling axis contributes to injury-induced nociceptive plasticity and the development of chronic pain. J Neurosci 37: 7481-7499.

15. Kim J, Salim M, Moy JK, Asiedu MN, Galo M, Vagner J, Price TJ (2016): Neuroligin 2 regulates spinal GABAergic plasticity in hyperalgesic priming, a model of the transition from acute to chronic pain. Pain 157: 1314-1324.

16. Kim JY V., Tillu D V., Quinn TL, Mejia GL, Shy A, Asiedu MNK, et al. (2015): Spinal dopaminergic projections control the transition to pathological pain plasticity via a D1/D5-mediated mechanism. $J$ Neurosci 35: 6307-6317.

17. Asiedu MN, Tillu D V., Melemedjian OK, Shy A, Sanoja R, Bodell B, et al. (2011): Spinal protein kinase $\mathrm{M} £$ underlies the maintenance mechanism of persistent nociceptive sensitization. $J$ Neurosci 31: 6646-6653.

18. Melemedjian OK, Asiedu MN, Tillu D V., Peebles KA, Yan J, Ertz N, et al. (2010): IL-6- and NGFinduced rapid control of protein synthesis and nociceptive plasticity via convergent signaling to the eIF4F complex. J Neurosci 30: 15113-15123.

19. Rivat C, Laboureyras E, Laulin JP, Le Roy C, Richebé P, Simonnet G (2007): Non-nociceptive environmental stress induces hyperalgesia, not analgesia, in pain and opioid-experienced rats. Neuropsychopharmacology. https://doi.org/10.1038/sj.npp.1301340 
20. Taylor B, Sinha G, Donahue R, Grachen C, Morón JA, Doolen S (2019): Opioid Receptors Inhibit the Spinal AMPA Receptor Ca2+ Permeability that Mediates Latent Pain Sensitization. Exp neurol 314: $58-66$.

21. Marvizon JC, Walwyn W, Minasyan A, Chen W, Taylor BK (2015): Latent sensitization: A model for stress-sensitive chronic pain. Curr Protoc Neurosci 2015: 9.50.1-9.50.15.

22. Custodio-Patsey L, Donahue RR, Fu W, Lambert J, Smith BN, Taylor BK (2020): Sex differences in kappa opioid receptor inhibition of latent postoperative pain sensitization in dorsal horn.

Neuropharmacology 163: 107726.

23. Maletic V, Raison CL (2009): Neurobiology of depression, fibromyalgia and neuropathic pain. Front Biosci. https://doi.org/10.2741/3598

24. Bair M, Robinson R, Katon W, Kroenke K (2003): Depression and Pain Comorbidity A Literature Review. Arch Intern Med 20: 2433-2445.

25. McWilliams LA, Goodwin RD, Cox BJ (2004): Depression and anxiety associated with three pain conditions: Results from a nationally representative sample. Pain 111: 77-83.

26. Simon G, VonKorff M, Piccinelli M, Fullerton C, Ormel J (1999): AN INTERNATIONAL STUDY OF THE RELATION BETWEEN SOMATIC SYMPTOMS AND DEPRESSION. new Engl J Med 341: 1329-1335.

27. Arntz A, Dreessen L, De Jong P (1994): The influence of anxiety on pain: attentional and attributional mediators. Pain 56: 307-314.

28. Ploghaus A, Narain C, Beckmann CF, Clare S, Bantick S, Wise R, et al. (2001): Exacerbation of pain by anxiety is associated with activity in a hippocampal network. J Neurosci 21: 9896-9903.

29. Dworkin, Robert H., Gitlin M (n.d.): Clinical_Aspects_of_Depression_in_Chronic_Pain.4.pdf.

30. Sheng J, Liu S, Wang Y, Cui R, Zhang X (2017): The Link between Depression and Chronic Pain: Neural Mechanisms in the Brain. Neural Plast 2017. https://doi.org/10.1155/2017/9724371

31. Rivat C, Sar C, Mechaly I, Leyris JP, Diouloufet L, Sonrier C, et al. (2018): Inhibition of neuronal FLT3 receptor tyrosine kinase alleviates peripheral neuropathic pain in mice. Nat Commun. https://doi.org/10.1038/s41467-018-03496-2

32. Brennan TJ, Vandermeulen EP, Gebhart GF (1996): Characterization of a rat model of incisional pain. Pain. https://doi.org/10.1016/0304-3959(95)01441-1

33. Eisch AJ, Petrik D (2012): Depression and hippocampal neurogenesis: A road to remission? Science (80- ) 338: 72-75.

34. Blugeot A, Rivat C, Bouvier E, Molet J, Mouchard A, Zeau B, et al. (2011): Vulnerability to depression: From brain neuroplasticity to identification of biomarkers. J Neurosci 31: 12889-12899.

35. Bouvier E, Brouillard F, Molet J, Claverie D, Cabungcal JH, Cresto N, et al. (2017): Nrf2-dependent persistent oxidative stress results in stress-induced vulnerability to depression. Mol Psychiatry 22: 1701-1713.

36. Cabañero D, Campillo A, Célérier E, Romero A, Puig MM (2009): Pronociceptive effects of remifentanil in a mouse model of postsurgical pain: Effect of a second surgery. Anesthesiology. https://doi.org/10.1097/ALN.0b013e3181bfab61

37. King T, Vera-Portocarrero L, Gutierrez T, Vanderah TW, Dussor G, Lai J, et al. (2009): Unmasking the tonic-aversive state in neuropathic pain. Nat Neurosci. https://doi.org/10.1038/nn.2407

38. Obata K, Yamanaka H, Fukuoka T, Yi D, Tokunaga A, Hashimoto N, et al. (2003): Contribution of injured and uninjured dorsal root ganglion neurons to pain behavior and the changes in gene expression following chronic constriction injury of the sciatic nerve in rats. Pain 101: 65-77.

39. Guan Z, Kuhn JA, Wang X, Colquitt B, Solorzano C, Vaman S, et al. (2015): Injured sensory neuronderived CSF1 induces microglial proliferation and DAP12-dependent pain. Nat Neurosci 19: 1-10.

40. Kobayashi K, Imagama S, Ohgomori T, Hirano K, Uchimura K, Sakamoto K, et al. (2013): Minocycline selectively inhibits M1 polarization of microglia. Cell Death Dis 4: 1-9.

41. Conway JG, McDonald B, Parham J, Keith B, Rusnak DW, Shaw E, et al. (2005): Inhibition of colony-stimulating-factor-1 signaling in vivo with the orally bioavailable cFMS kinase inhibitor 
GW2580. Proc Natl Acad Sci U S A 102: 16078-16083.

42. Mills SEE, Nicolson KP, Smith BH (2019): Chronic pain: a review of its epidemiology and associated factors in population-based studies. Br J Anaesth. https://doi.org/10.1016/j.bja.2019.03.023

43. Rivat C, Laulin J-P, Corcuff J-B, Célèrier E, Pain L, Simonnet G (2002): Fentanyl enhancement of carrageenan-induced long-lasting hyperalgesia in rats: prevention by the N-methyl-D-aspartate receptor antagonist ketamine. Anesthesiology 96: 381-391.

44. Sheline YI, Sanghavi M, Mintun MA, Gado MH (1999): Depression duration but not age predicts hippocampal volume loss in medically healthy women with recurrent major depression. $J$ Neurosci.

45. Bremner JD, Narayan M, Anderson ER, Staib LH, Miller HL, Charney DS (2000): Hippocampal volume reduction in major depression. Am J Psychiatry. https://doi.org/10.1176/ajp.157.1.115

46. Duman RS, Monteggia LM (2006): A Neurotrophic Model for Stress-Related Mood Disorders. Biological Psychiatry. https://doi.org/10.1016/j.biopsych.2006.02.013

47. Pittenger C, Duman RS (2008): Stress, depression, and neuroplasticity: A convergence of mechanisms. Neuropsychopharmacology. https://doi.org/10.1038/sj.npp.1301574

48. Suzuki T, Amata M, Sakaue G, Nishimura S, Inoue T, Shibata M, Mashimo T (2007): Experimental neuropathy in mice is associated with delayed behavioral changes related to anxiety and depression. Anesth Analg. https://doi.org/10.1213/01.ane.0000261514.19946.66

49. Gonçalves L, Silva R, Pinto-Ribeiro F, Pêgo JM, Bessa JM, Pertovaara A, et al. (2008): Neuropathic pain is associated with depressive behaviour and induces neuroplasticity in the amygdala of the rat. Exp Neurol. https://doi.org/10.1016/j.expneurol.2008.04.043

50. Yalcin I, Bohren Y, Waltisperger E, Sage-Ciocca D, Yin JC, Freund-Mercier MJ, Barrot M (2011): A time-dependent history of mood disorders in a murine model of neuropathic pain. Biol Psychiatry. https://doi.org/10.1016/j.biopsych.2011.07.017

51. Zhou W, Jin Y, Meng Q, Zhu X, Bai T, Tian Y, et al. (2019): A neural circuit for comorbid depressive symptoms in chronic pain. Nat Neurosci 22. https://doi.org/10.1038/s41593-019-0468-2

52. Becker LJ, Journée SH, Lutz PE, Yalcin I (2020): Comorbidity of chronic pain and anxiodepressive disorders: Deciphering underlying brain circuits. Neurosci Biobehav Rev 115: 131-133.

53. Schwaller F, Beggs S, Walker SM (2015): Targeting p38 Mitogen-activated Protein Kinase to Reduce the Impact of Neonatal Microglial Priming on Incision-induced Hyperalgesia in the Adult Rat. Anesthesiology 122: 1377-1390.

54. Obata H, Eisenach JC, Hussain H, Bynum T, Vincler M (2006): Spinal Glial Activation Contributes to Postoperative Mechanical Hypersensitivity in the Rat. J Pain. https://doi.org/10.1016/j.jpain.2006.04.004

55. Okubo M, Yamanaka H, Kobayashi K, Dai Y, Kanda H, Yagi H, Noguchi K (2016): MacrophageColony Stimulating Factor Derived from Injured Primary Afferent Induces Proliferation of Spinal Microglia and Neuropathic Pain in Rats. PLoS One. https://doi.org/10.1371/journal.pone.0153375

56. Méchaly I, Bourane S, Piquemal D, Al-Jumaily M, Ventéo S, Puech S, et al. (2006): Gene profiling during development and after a peripheral nerve traumatism reveals genes specifically induced by injury in dorsal root ganglia. Mol Cell Neurosci 32: 217-229.

57. Haroutiunian S, Nikolajsen L, Finnerup NB, Jensen TS (2013): The neuropathic component in persistent postsurgical pain: A systematic literature review. Pain. https://doi.org/10.1016/j.pain.2012.09.010

58. Robin G, Martineau P (n.d.): Synthetic Customized ScFv Libraries. Methods Mol Biol 907, 109-22 (2012).

59. Robin G, Sato Y, Desplancq D, Rochel N, Weiss E, Martineau P (2014): Restricted diversity of antigen binding residues of antibodies revealed by computational alanine scanning of 227 antibodyantigen complexes. J Mol Biol 426: 3729-3743. 


\section{Figure Legends}

Figure 1: Influence of single (SI) or double incision (DI) on emotional related behaviors and neurogenesis. During the second week after surgery (Sham, SI, DI), (A) decreased grooming behavior in the splash test, (B) increased latency to eat in the novelty suppressed feeding test and (C) increased immobility duration in the forced swim test is observed only in DI mice compared to control mice. (D) Summary table of anxiodepressive-like behaviors in all tests at three different time points. (E) Brdu/NeuN positive nuclei per section in the dentate gyrus is significantly reduced only in DI mice 7 days post-injury (scale bars $=200 \mu \mathrm{m}$ ) and $(\mathbf{F})$ related quantification. All the values are means \pm s.e.m. ( $\mathrm{n}=12$ except in $\mathbf{E}, \mathbf{F}, \mathrm{n}=4)$. One-way ANOVA and Holm-Sidak's test $(\mathbf{A}, \mathbf{B}, \mathbf{C})$ or Kruskal-Wallis test $(\mathbf{F}) ;{ }^{*} \mathrm{P}<0.05 ; * * \mathrm{P}<0.01 ; * * \mathrm{P}<0.001$ vs. Sham.

Figure 2: Influence of single (SI) or double (DI) incision on sensorial behaviors and microgliosis. (A) Mechanical hypersensitivity after incisions as measured by the Von Frey test and (B) cumulative area under curve representation show increased mechanical hypersensitivity after DI. (C) Cumulative area under curve representation of Hargreave's test shows increased thermal hypersensitivity after DI. (D) Conditioned place preference induced by clonidine-evoked analgesia 4 days post-Sham, SI or DI. (E) Changes in spinal microgliosis related biomarkers mRNA expression as measured with RT-qPCR 3 days after Sham, SI or DI. (F) Iba1 immunoreactivity in the whole spinal cord (left panel) and zoomed dorsal horn (right panel) 7 days after Sham, SI or DI (scale bars $=200 \mu \mathrm{m})$ and $(\mathbf{G})$ related quantification. $(\mathbf{H})$ Mechanical threshold of Sham, SI and DI animals before (3 days post-procedure) and 15 minutes after intrathecal injection of minocycline (300 $\mu \mathrm{g} /$ mouse). All the values are means \pm s.e.m. ( $\mathrm{n}=8$ except in $\mathbf{D}, \mathrm{n}=10$ and $\mathbf{E}-\mathbf{G}, \mathrm{n}=4)$. Two- 
way ANOVA and Holm-Sidak's test (A); Student's t-test (B-C); One-way ANOVA and HolmSidak's test $(\mathbf{D}-\mathbf{H}) ;{ }^{*} \mathrm{P}<0.05 ; * * \mathrm{P}<0.01 ; * * * \mathrm{P}<0.001$ vs. Sham, baseline or vehicle paired; $\# \mathrm{P}<0.05$; $\# \# \mathrm{P}<0.01 ; \# \# \# \mathrm{P}<0.001$ vs. SI.

Figure 3: Silencing Flt3 expression blocks DI-induced behavioral and molecular sensitization. (A, B) Left and right hindpaw mechanical hypersensitivity after incisions on $\mathrm{Flt}^{+/+}$or $\mathrm{Flt}^{-/-}$mice as measured by the Von Frey test and $(\mathbf{C})$ cumulative area under curve representation highlight the absence of mechanical hypersensitivity exaggeration after DI in $\mathrm{Flt3}^{-/}$. (D) Conditioned place preference induced by clonidine-evoked analgesia 4 days post-injury is totally prevented in $\mathrm{Flt}^{-/}$. Silencing Flt3 expression did not change locomotor activity (E) and latency to eat in novelty suppressed feeding test $(\mathbf{G})$, but increases grooming duration in splash test $(\mathbf{F})$ and decreases immobility duration in forced swim test $(\mathbf{H})$ compared to $F l t 3^{+/+}$mice. (I) Iba1 immunoreactivity of spinal cord left dorsal horn dissected from Sham or DI $\left(F l t 3^{-/-}\right.$or control) mice and $(\mathbf{J})$ related quantification show significantly higher labeling density in DI Flt ${ }^{+/+}$mice compared to Sham $\mathrm{Flt}^{+/+}$. (K) Ibal immunoreactivity of spinal cord right dorsal horn dissected from Sham or DI (Flt $3^{-/}$or control) mice and (L) related quantification show significantly higher labeling density in DI Flt $3^{+/+}$mice compared to Sham $\mathrm{Flt}^{+/+}$. All the values are means \pm s.e.m. $(\mathrm{n}=8 / 9$ except in $\mathbf{I}-\mathbf{L}$, n=6/7). Two-way ANOVA and Holm-Sidak's test (A-B); Student's t-test (E-H); One-way ANOVA and Holm-Sidak's test $(\mathbf{C}, \mathbf{D}, \mathbf{J}, \mathbf{L}) ;{ }^{*} \mathrm{P}<0.05 ; * * \mathrm{P}<0.01 ; * * * \mathrm{P}<0.001 \mathrm{vs} . \mathrm{Flt}^{+/+}$or Sham $\mathrm{Flt3}^{+/+}$or Sham $\mathrm{Flt3}^{-/}$. 
Figure 4: FL administration mimics DI induced behavioral and molecular alterations. (A) Mechanical hypersensitivity measures with Von Frey filaments after a single intrathecal injection of FL $(50 \mathrm{ng} / 5 \mu \mathrm{l})$ or saline. (B) Repeated intrathecal injections of FL $(50 \mathrm{ng} / 5 \mu 1,5$ injections delivered across 15 days) fail to affect performance on rotarod, $(\mathbf{C})$ decrease grooming duration in splash test, (D) increase latency to eat in novelty suppressed feeding test and (E) immobility duration in forced swim test compared to control injections. (F) Iba1 immunoreactivity of whole spinal cord dissected from saline injected and FL injected mice $(50 \mathrm{ng} / 5 \mu 1$, daily injection for 3 days) 24 hours after the last injection (left scale bars $=200 \mu \mathrm{m}$; right scale bars $=20 \mu \mathrm{m}$ ) and (G) related quantification. All the values are means \pm s.e.m. ( $n=10$ except in $\mathbf{F}, \mathbf{G}, \mathrm{n}=4)$. Student's t-test (A-E); unpaired Mann-Whitney t-test $(\mathbf{G}) ;{ }^{*} \mathrm{P}<0.05 ; * * * \mathrm{P}<0.001$ vs. I.t. Vehicle.

Figure 5: MAbA3 is a high affinity functional antibody against both human and murine FLT3 receptor. Binding curves of FL on human (A) and murine FLT3 (B) in presence of increasing doses of mAbA3. MAbA3 reduces in a dose dependent manner HTRF signal reporting mouse FLT3 constitutive activity (C) and FL-induced increased FLT3 activity after FL application at 1 $\mathrm{nM}(\mathbf{D})$ and $10 \mathrm{nM}(\mathbf{E})$. All the values are means \pm s.e.m. $(\mathrm{n}=4) ; * \mathrm{P}<0.05 ; * * \mathrm{P}<0.01 ; * * * \mathrm{P}<0.001$ vs. Baseline

Figure 6: Functional antibodies treatment against FLT3 prevents the development of CPSP. (A) A single injection of mAbA3 $(100 \mu \mathrm{g} /$ mouse, intraperitoneal) reduces CCI-related (9 days after chronic constriction injury of the sciatic nerve) mechanical hypersensitivity as measured 1, 1.5 and 2 days after injection in the Von Frey test. (B) Experiments were performed as in A, with doses of 
mAbA3 ranging from 2 to $200 \mu \mathrm{g} /$ animal. Data represent the maximum percentage of pain

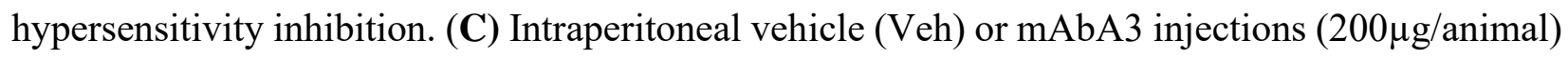
were initiated 25 days after CCI of the sciatic nerve and repeated at times indicated by arrows. Mechanical hypersensitivity was recorded 26, 28, 33, 35 and 44 days after CCI. (D-E) Left and right hindpaw mechanical hypersensitivity after incisions in either vehicle or mAbA3 treated (arrows $=200 \mu \mathrm{g} /$ animal, intraperitoneal) mice as measured by the Von Frey test. Repeated intraperitoneal injections of mAbA3 $(200 \mu \mathrm{g} /$ animal, 1 injection each 2 days during the first incision phase for a total of 4 injections) increases grooming duration in splash test $(\mathbf{F})$, decreases latency to eat in novelty suppressed feeding test $(\mathbf{G})$ and immobility duration in forced swim test $(\mathbf{H})$ in treated mice compared to control mice. Two-way ANOVA and Holm Sidak's test (A-E), Student's t-test $(\mathbf{F}-\mathbf{H}) ;{ }^{*} \mathrm{P}<0.05 ; * * \mathrm{P}<0.01 ; * * * \mathrm{P}<0.001$ vs. Sham-Veh or Vehicle. 
A

B

NSF

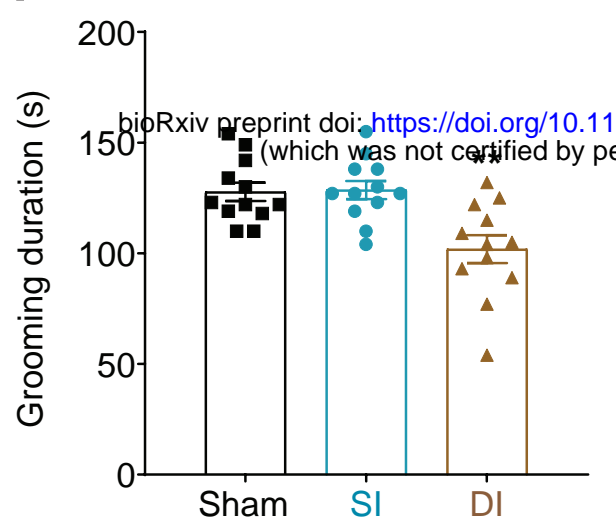

400

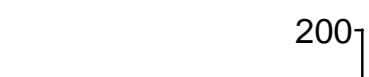

200

***

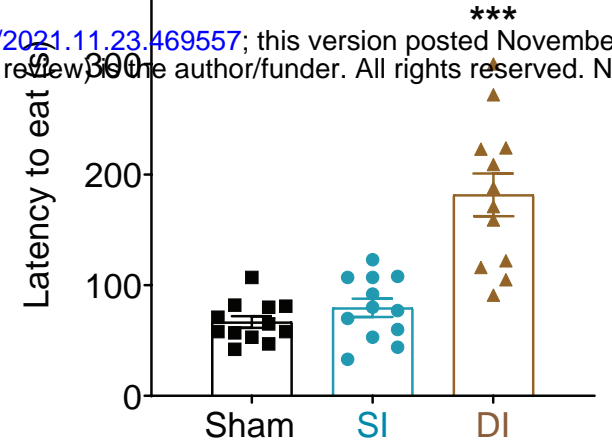

ชิ201. The copyright holder for this preprint

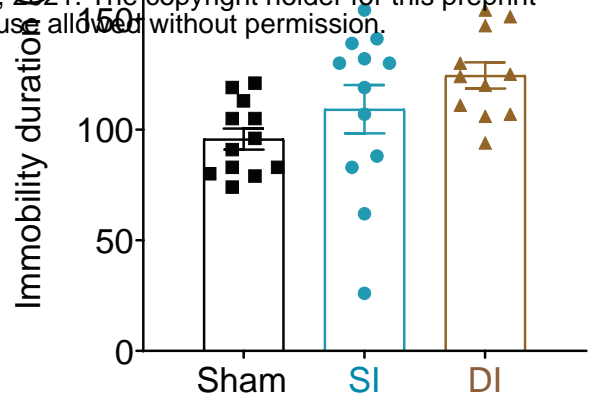

D

\begin{tabular}{|c|c|c|c|c|}
\hline \multirow{4}{*}{ SI } & Tests & $1^{\text {st }}$ week & $2^{\text {nd }}$ week & $4-5^{\text {th }}$ week \\
\hline & ST & - & - & - \\
\hline & NSF & + & - & - \\
\hline & FST & - & - & - \\
\hline
\end{tabular}

\begin{tabular}{|c|c|c|c|c|}
\hline \multirow{2}{*}{ DI } & Tests & $1^{\text {st }}$ week & $2^{\text {nd }}$ week & $4^{-5^{\text {th }}}$ week \\
\cline { 2 - 5 } & ST & + & + & - \\
\cline { 2 - 5 } & NSF & + & + & - \\
\cline { 2 - 5 } & FST & + & + & - \\
\hline
\end{tabular}

Recovery

E

F
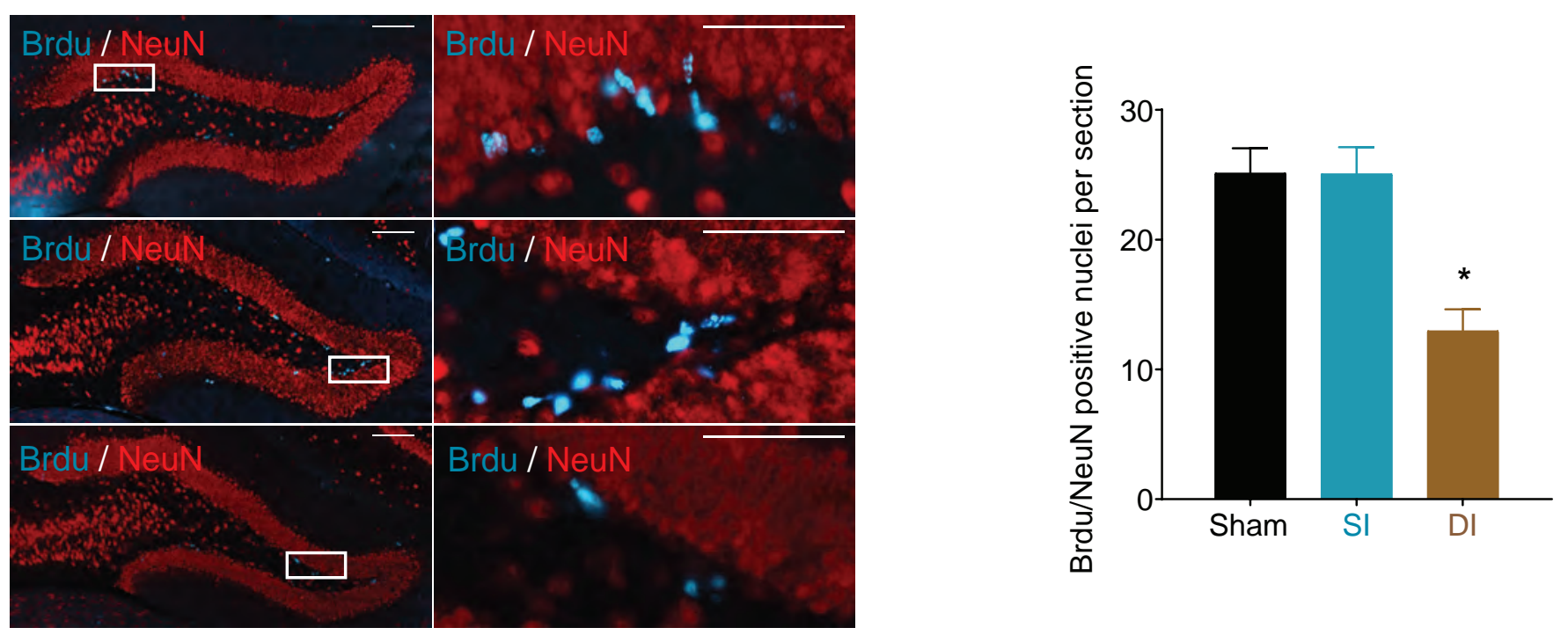

Figure 1 
A


F
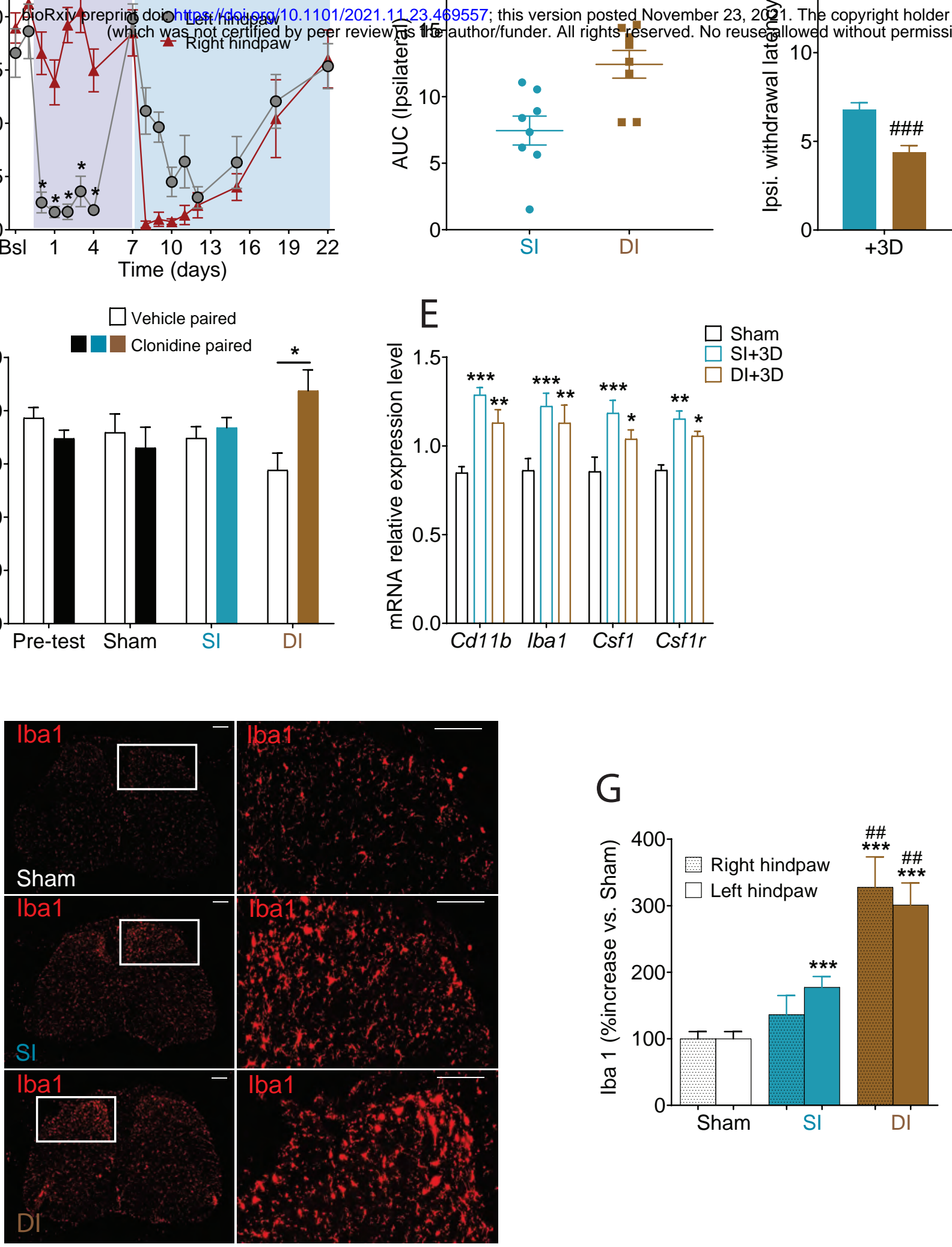

(n) $^{15}$
\#\#

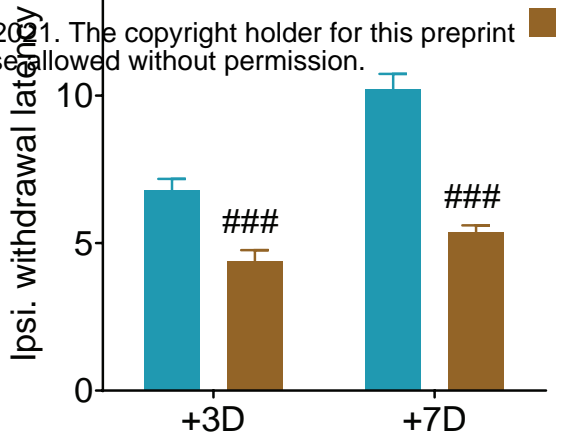

DI 
A Left hindpaw
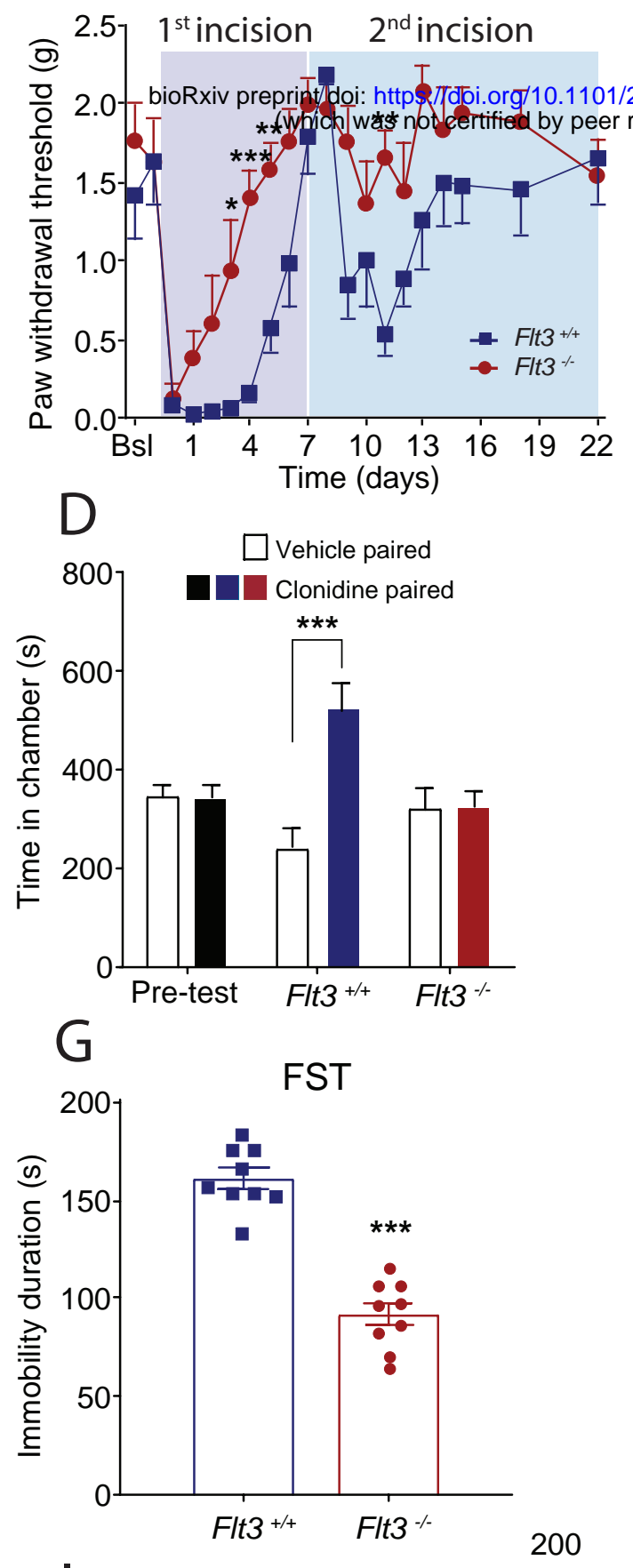

Left DH

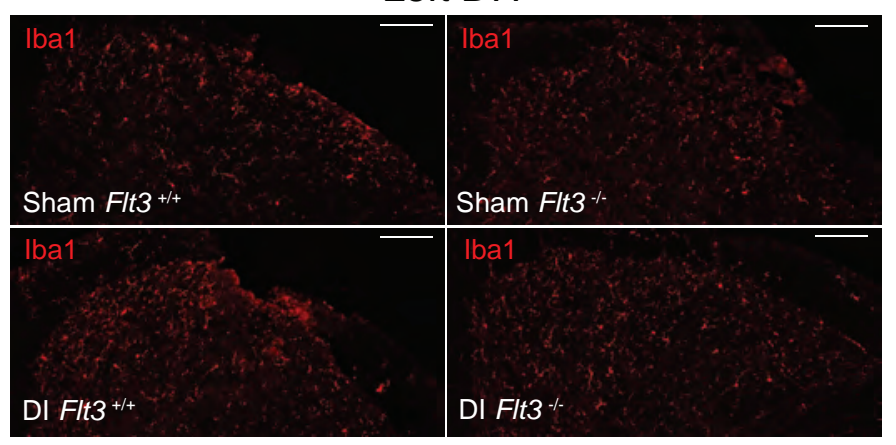

K

Right DH

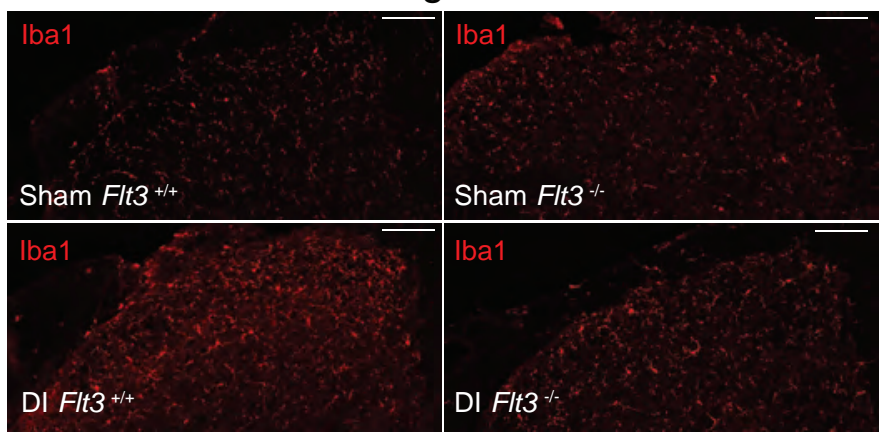

Right hindpaw

${ }^{2}{ }^{2.5} \cdot 1^{\text {st }}$ incision $2^{\text {nd }}$ incision

20

- Flt3 ${ }^{+/+}$

Flt3 $\%$
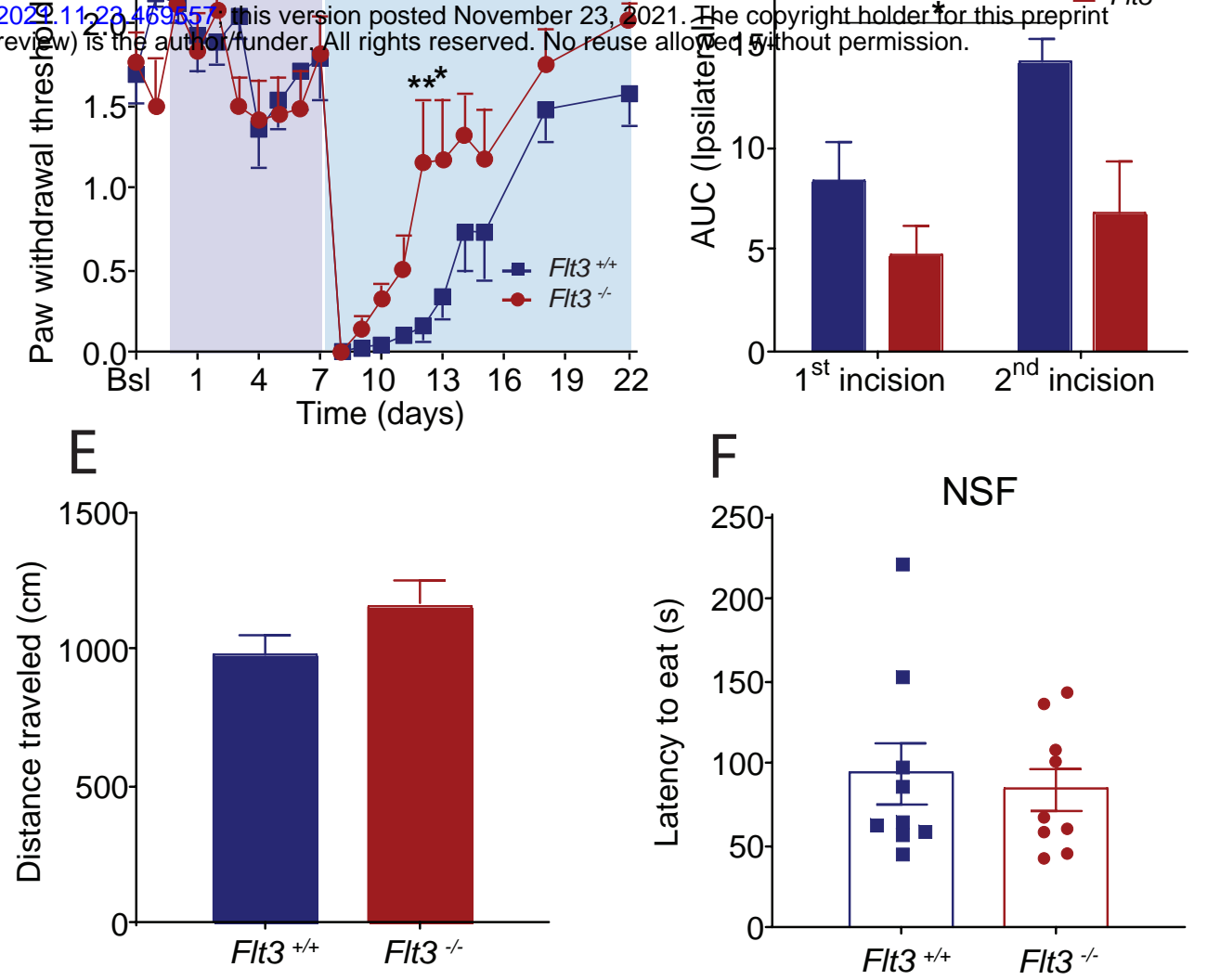
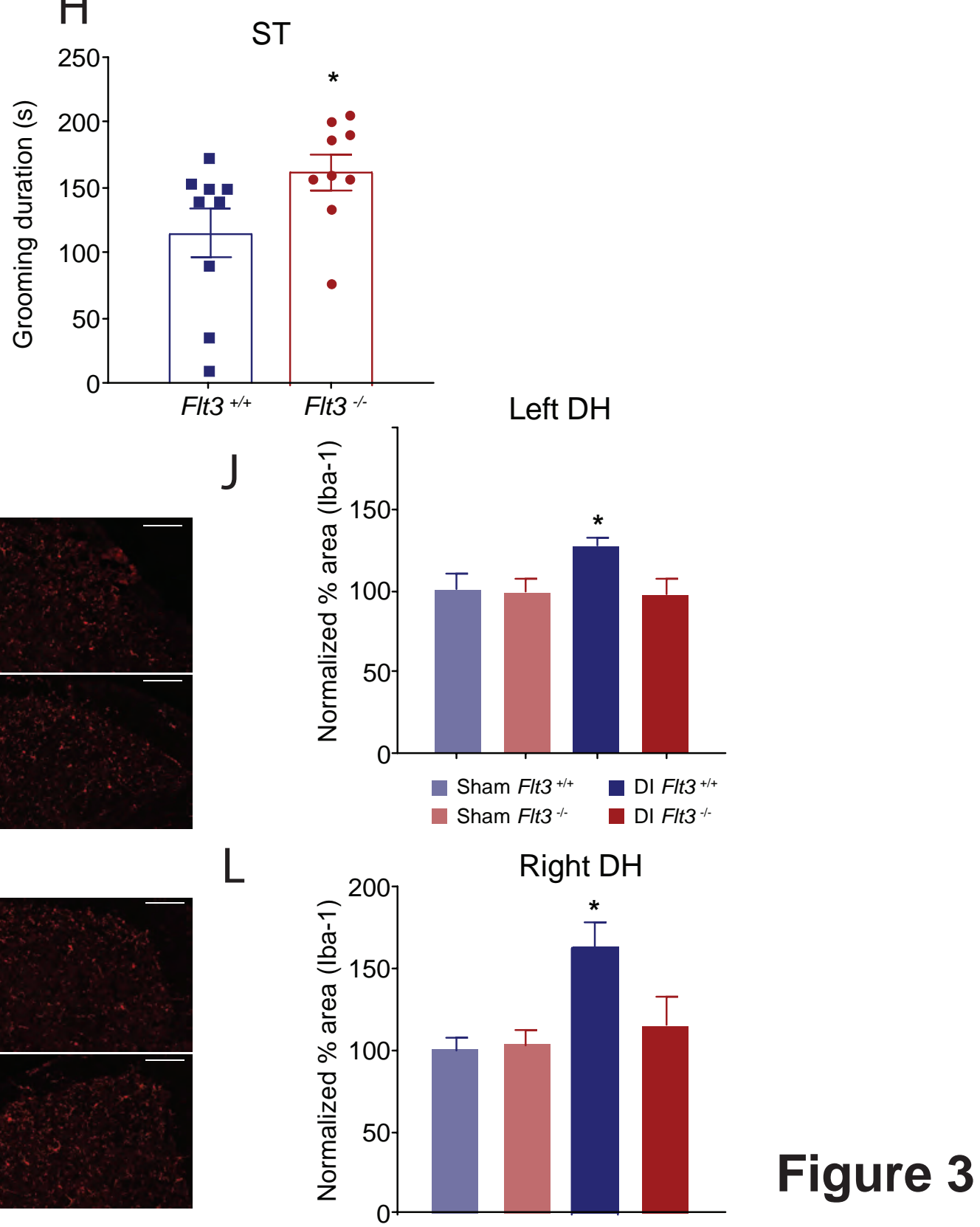


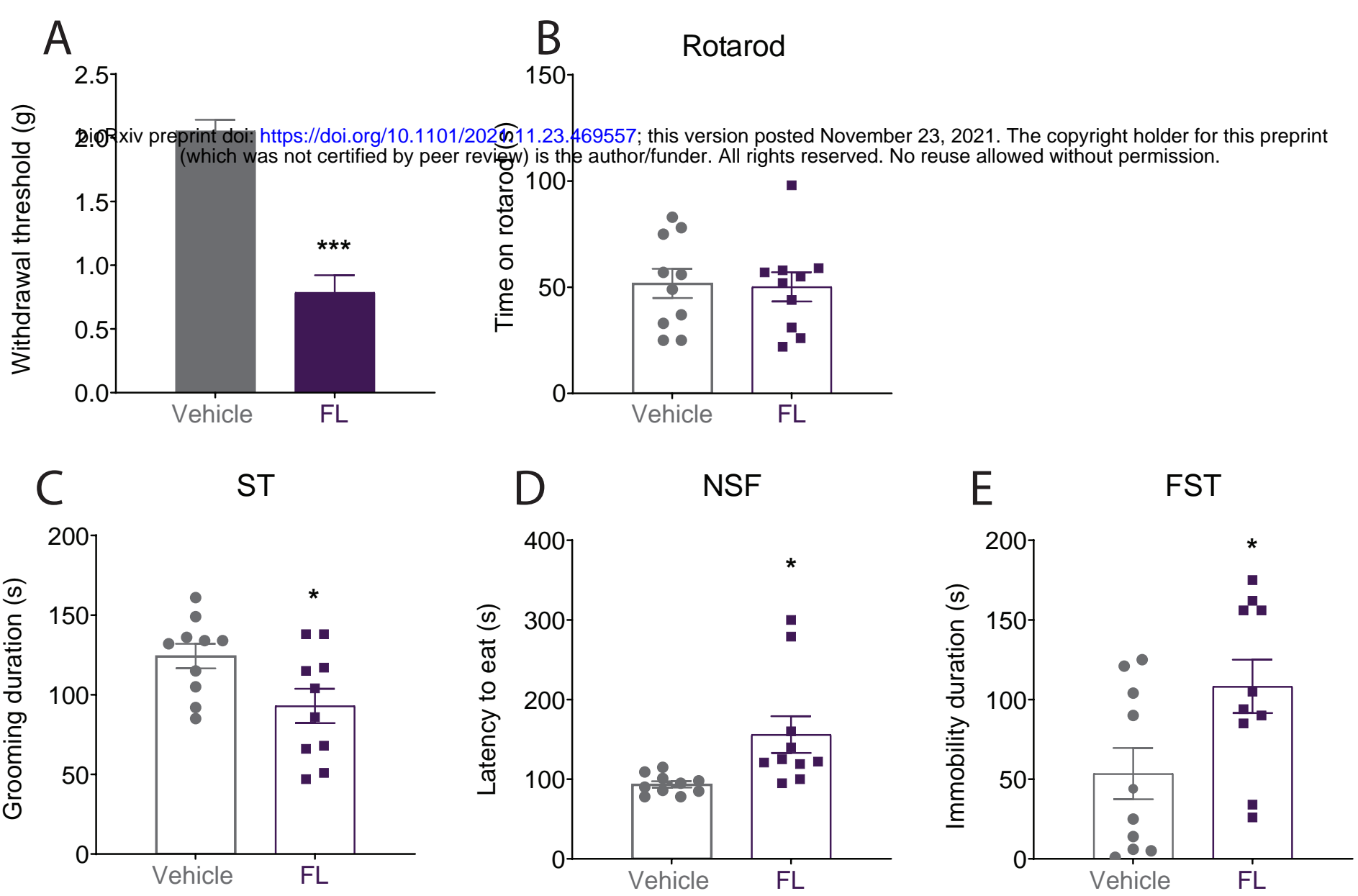

$\mathrm{F}$
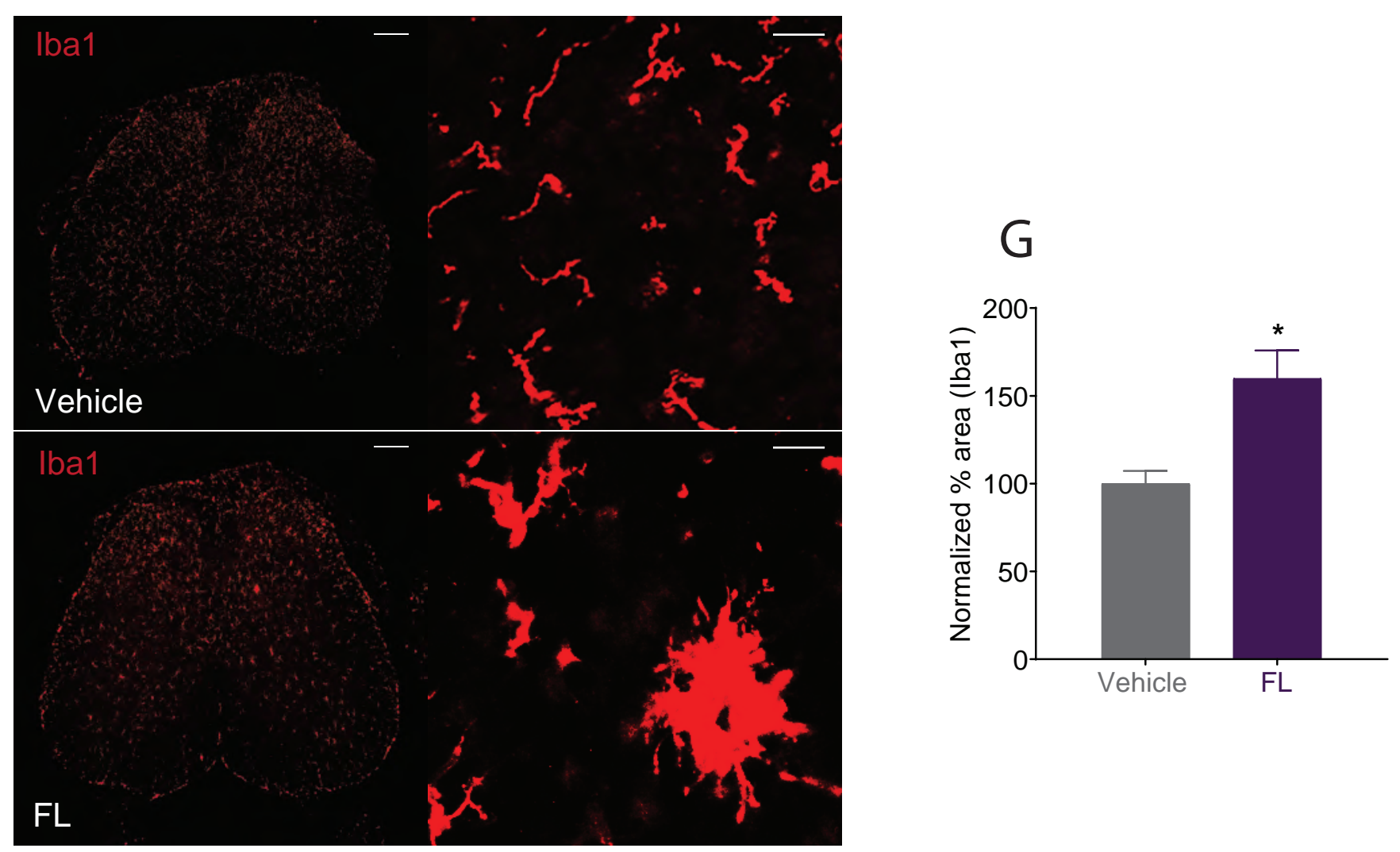
bioRxiv preprinfdoi: https://doi.organ 10.1101/2021.11.23.469557; this version p9gted November 23, 2021. The copyright holder for this preprint

bioRxiv preprinfdoi: https://doi.organ 10.1101/2021.11.23.469557; this version p9gted November 23, 2021. The copyright holder for this preprint
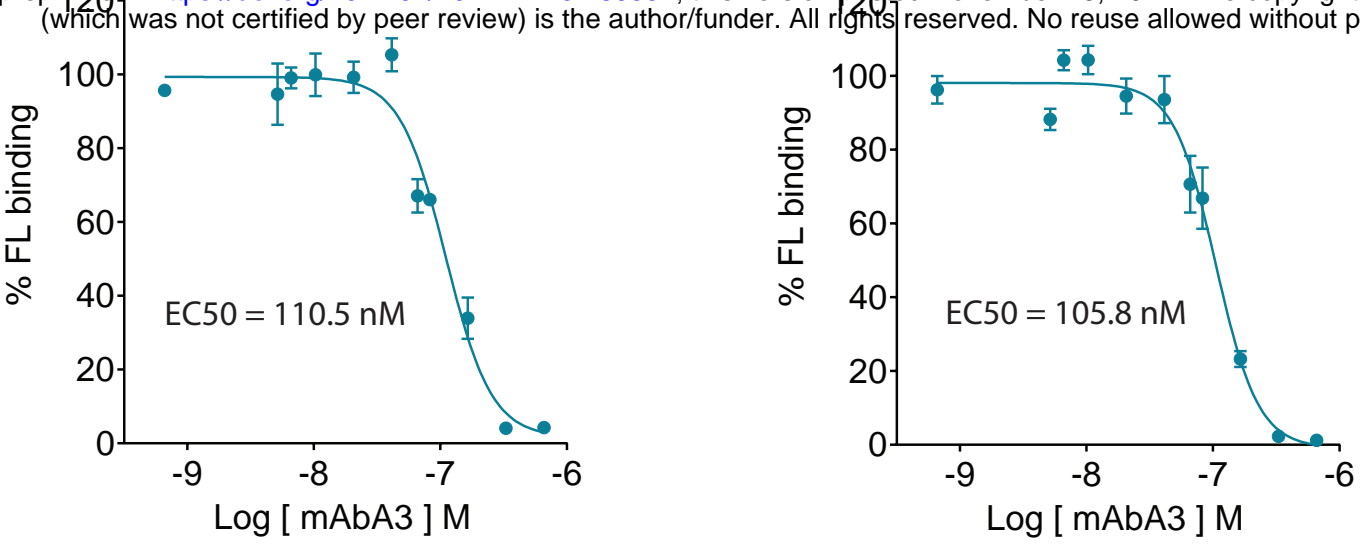

C

D

$\mathrm{FL}=1 \mathrm{nM}$

$\mathrm{E}$

$\mathrm{FL}=10 \mathrm{nM}$
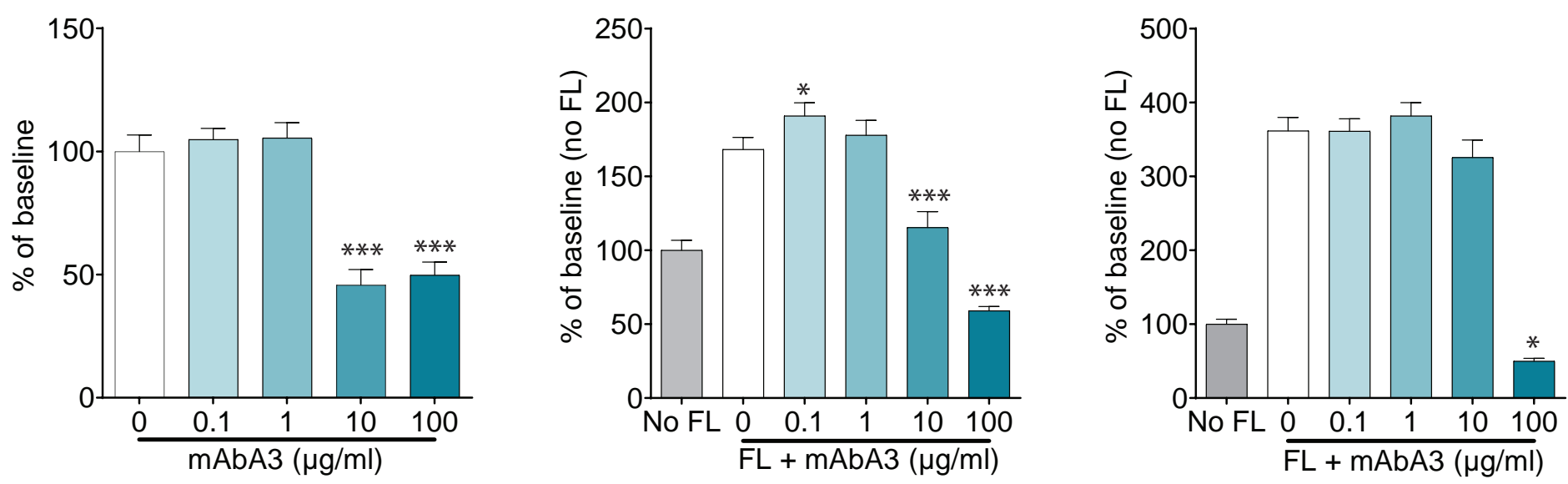

Figure 5 
